\title{
Post traumatic extracranial vertebral artery dissection with locked-in syndrome: a case with MRI documentation and unusually favourable outcome
}

\author{
A D RAE-GRANT, F LIN, B A YAEGER, P BARBOUR, L P LEVITT, J E CASTALDO, \\ M C LESTER \\ From the Division of Neurology, Lehigh Valley Hospital Center, Allentown, PA, USA
}

SUMMARY A patient with a right vertebral artery dissection occurring after minor head trauma progressed to a massive pontine infarction. An unusually favourable outcome and MRI imaging are discussed.

Post-traumatic extracranial vertebral artery dissections are rare events and often difficult to diagnose. MRI scanning has been shown to be extraordinarily sensitive in detecting vascular brain injury, especially in posterior circulation vascular disease. Whereas pontine lesions are poorly imaged using CT scanning techniques, these areas are exquisitely accessible to this imaging modality.

We report a case of post-traumatic extracranial vertebral dissection resulting in a large pontine haemorrhagic infarction, imaged with MRI, in which a severe locked-in state had an unusually favourable outcome.

\section{Case report}

A 31 year old right handed white male was involved in a motor vehicle accident and suffered a minor concussion. Two weeks later he developed a persistent right posterior neck and occiput headache, associated with nausea and vomiting. After two days the patient developed a right lower motor neuron facial paresis and was admitted to another hospital. Over a period of five hours he became lethargic and developed dysarthria and a progressive left hemiparesis. He was then transferred to our institution. Within hours he developed a quadriparesis, facial diplegia and loss of horizontal eye movements. He was fully alert and answered complex questions by looking up or down. Pupils were $2 \mathrm{~mm}$ and reactive, up and down eye movements were present but slowed. Corneal reflexes were reduced. Gag was absent.

Correspondence to: Dr Rae-Grant, 1210 S Cedar Crest Boulevard, Allentown, PA 18103, USA.

Received 14 February 1989.

Accepted 24 May 1989
There was minimal flexion of the left arm to pain, with no motion of the other limbs. Deep tendon reflexes were pathologically brisk in four limbs with clonus and positive Babinski signs present.

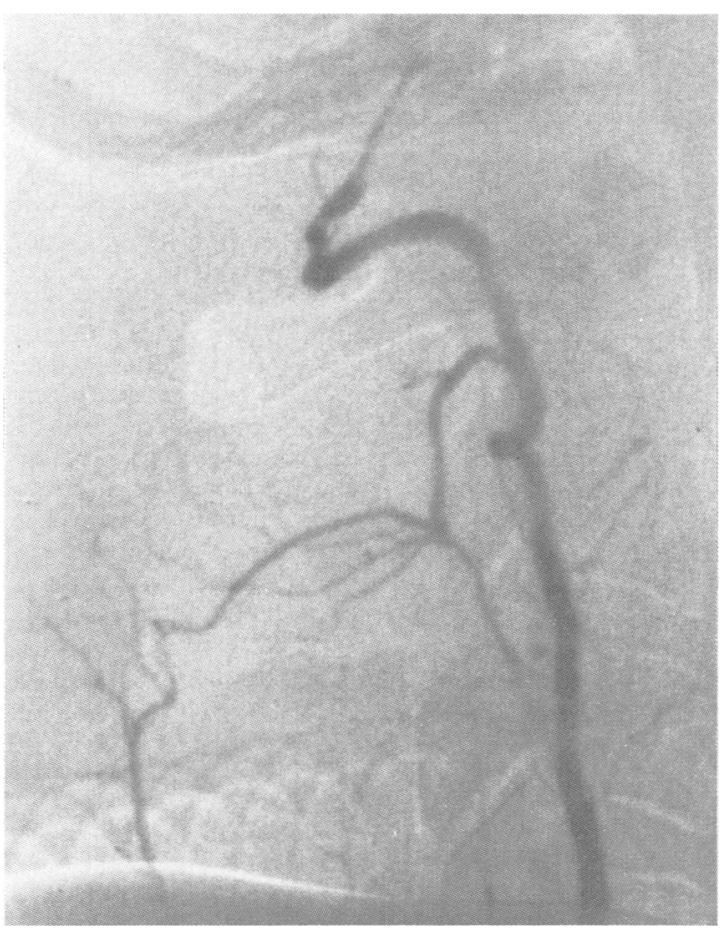

Fig 1 Selective right vertebral angiogram showing an irregular tapering occlusion at the C1-2 level. 

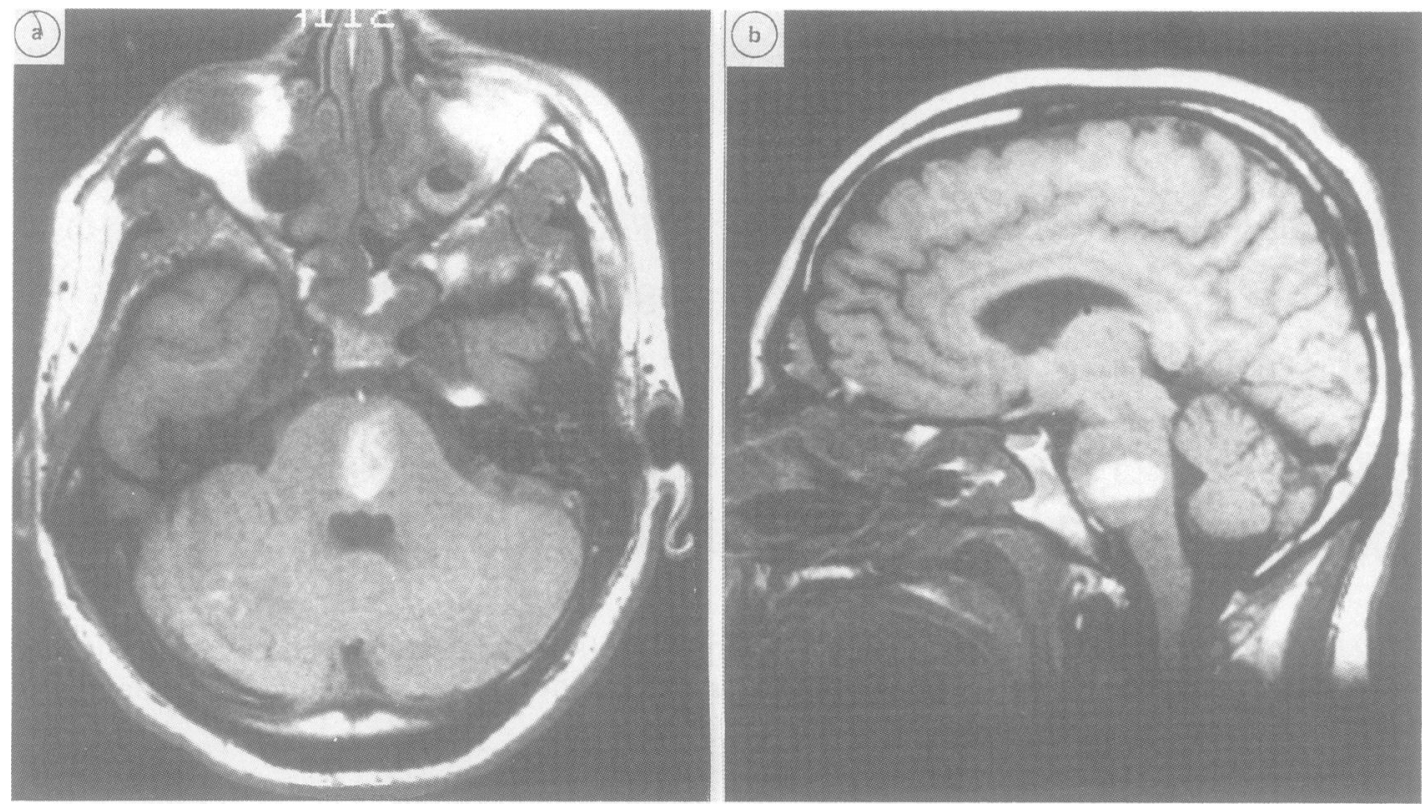

Fig 2a Axial MRI scans of the brainstem with TR 700, TE 20, showing haemorrhagic infarction in the pons.

Fig $2 \mathrm{~b}$ Sagittal MRI scans of the brainstem with TR 700, TE 20, showing haemorrhagic infarction in the pons.

Initial CT scan of the head showed an area of lucency in the right cerebellum, but no other abnormalities. Cerebral angiography showed an irregular tapering occlusion of the right extracranial vertebral artery at the C1-2 level (fig 1), with lack of flow in the basilar artery. The left vertebral artery terminated as a posterior inferior cerebellar artery. There was reconstitution of the tip of the basilar from the posterior communicating arteries on carotid injection.

Magnetic resonance imaging (MRI) was obtained one week after admission on a clinical 1.5 Tesla system (Signa, GE). Both spin echo and gradient recalled echo techniques were employed. The pons was diffusely enlarged and nearly contiguous with the clivus. Within the pons, there was a $1 \cdot 1$ $\times 1.3 \times 2.1$ centimetre region of considerably increased signal intensity on the short TR images. This region represented methemoglobin formation from a subacute haemorrhage (fig 2). On the long TR images there were irregular regions in the pons of increased signal intensity consistent with areas of infarction. Only the most lateral aspect of the pons as well as the posterior superior portion appeared spared. The medulla and midbrain appeared spared. There was an irregular region of increased signal intensity on the long TR images within the right cerebellar hemisphere consistent with infarction. Flow was documented within both proximal vertebral arteries; however, no flow could be identified within the basilar artery. The posterior cerebral arteries were patent. The supratentorial structures were within normal limits.

Over a three month period the patient progressively improved. He regained slow and incomplete lateral eye movements, head nodding, slow facial movements and mildly paretic movements of all limbs. He was able to communicate using a computer and was learning to walk again.

\section{Discussion}

This case highlights a number of points on extracranial vertebral dissection. There was a significant delay between the minor head injury and the time of dissection. Many dissections of the extracranial vertebral artery are spontaneous, though some are preceded by minor neck trauma or manipulation. ${ }^{1-3}$ Symptoms of extracranial vertebral dissections may occur at anytime, from minutes to days after trauma. ${ }^{4}$ In some cases, the delay may be extended up to six weeks, which may confound the diagnosis. ${ }^{1}$ This patient had the cardinal symptoms of vertebral dissection: neck and headache, nausea, vomiting and progressive brainstem signs. The presence of dissection was suggested by the irregular tapered narrowing of the extracranial vertebral arteries. An early tear may have been extended by subsequent minor injury from neck turning or other unnoticed minor injury. Dissections have been associated with fibromuscular dysplasia, migraine, chronic hypertension and oral contraceptive use in anecdotal reports ${ }^{25}$; none of these aetiologies were apparent in this case.

This MRI appearance with haemorrhagic infarction in the pons has not previously been described with this 
aetiology. MRI is undoubtedly useful in the diagnosis of basilar thrombosis, especially in early cases. ${ }^{367}$ The earliest sign is a flow-void phenomenon with linear structure on $\mathrm{T} 1$ weighted parasagittal scans and loss of the normal T2 flow-void seen in the basilar artery. Later changes include hypointense areas on $\mathrm{Tl}$ weighted images, and hyperintense $\mathrm{T} 2$ images. All of these signs were present in this case. In addition, there was evidence of haemorrhagic infarction with methemoglobin deposition on the MRI.

Most dissections of the extracranial vertebral artery show signs of unilateral low brainstem ischaemia only; these patients have a relatively benign course. In those cases with pontine infarction, recovery is usually poor. ${ }^{135}$ This patient showed an unusual recovery considering the extent of infarction. This may have been due to his young age and aggressive supportive measures. Because of the advantages in imaging with MRI, our understanding of the syndromes associated with vertebral dissections will undoubtedly improve.

\section{References}

1 Mas JL, Bousser MG, Hasboun D. Laplane D. Extracranial vertebral artery dissections: a review of 13 cases. Stroke 1987;18:1037-947.

2 Caplan LR, Zarins CK, Hemmati M. Spontaneous dissection of the extracranial vertebral arteries. Stroke 1985;16:1030-8.

3 Povlsen UJ, Kjaer L, Arlien-Soborg P. Locked-in syndrome following cervical manipulation. Acta Neurol Scand 1987;76:486-8.

4 Friedman AH. Arterial dissections. In: Wilkins RH, Rengachary SS, eds. Neurosurgery. New York: McGraw-Hill, 1985:1297-300.

5 Mokri B, Houser OW, Sandok BA, Piepgras DG. Spontaneous dissections of the vertebral arteries. Neurology 1988;38:880-5.

6 Biller J, Yuh WTC, Mitchell GW, et al. Early diagnosis of basilar artery occlusion using magnetic resonance imaging. Stroke 1988;19:297-306.

7 Baker RS, Carr WA. Pontine infarction: angiography and magnetic fresonance imaging. Surv Ophthalmol 1987;32(4):141-3. 